\title{
Forecasting method of electric vehicle load time-space distribution considering traffic distribution
}

\author{
Weidong Liu ${ }^{1}$, Lei $\mathrm{Li}^{1 *}$, Qin $\mathrm{Xie}^{2}$, Dan $\mathrm{Li}^{1}$ and Jing Zhang ${ }^{3}$ \\ ${ }^{1}$ Marketing Service Center of State Grid Tianjin Electric Power Company, Tianjin, 300202, China \\ ${ }^{2}$ State Grid Tianjin Electric Power Company, Tianjin, 300010, China \\ ${ }^{3}$ China Electric Power Research Institute, Beijing, 100192, China \\ *Corresponding author's e-mail: lei.li@tj.sgcc.com.cn
}

\begin{abstract}
The main work of this paper is to establish an electric vehicle(EV) load forecasting model based on road network traffic distribution for urban and inter-city transportation networks. This paper established a road network model considering the traffic impedance for the EV load forecasting of the urban fast charging network, and studied the prediction method of the time-space distribution of EV charging demand in the fast charging mode .Based on the expressway, the method for predicting the time-space distribution of EV load in the inter-city fast charging network is studied, and a time-space distribution load forecasting model is established. Based on the time-space distribution of traffic flow, combined with EV charging characteristics and travel routes, load simulation is performed. By constructing a prediction method for the time-space distribution of EV charging demand in the fast charging mode, it provides theoretical and methodological support for the research of time-sharing and segmented metering and charging strategies for EV fast charging stations, and provides an important reference for the development of EV charging facilities operating cost benefits, economic performance indicators and calculation models under fast charging mode, which are of great significance to promote the popularization and application of EV fast charging modes.
\end{abstract}

\section{Introduction}

With the gradual implementation of the strategic policy of vigorously promoting the large-scale development of EVs, the gradual maturation and deepening of the application of EVs and their fast charging technologies, EV urban public fast charging networks and intercity interconnected fast charging networks are gradually taking shape, but charging facilities it is not compatible with the existing operating model and its development model.

The current research on the prediction of the timespace distribution characteristics of the conventional charging demand for EVs has obtained some theoretical methods, most of which are charged in residential areas, or in the scene of commercial vehicles such as public transportation sanitation vehicles, which are mainly used for power exchange. Research on the time-space distribution characteristics of fast charging demand in urban public areas.Han Seung studied the coordination of charging for EVs: reducing peak loads and minimizing charging costs[1]; Xun Zong studied a neural dynamics algorithm for optimizing the residential demand response of plug-in EVs[2], Jing Zhang studied the population of vehicle users daily EV charging load curve based on statistical data[3]; M. Kandidayeni studies the influence of fuel cell system aging and thermal management on energy management strategies[4]; Veeraj designed an EV battery pack thermal management system[5].However, none of them proposed methods for predicting the time-space distribution of EV load in consideration of traffic distribution.

Carrying out the research of EV charging load forecasting method under fast charging mode can provide a theoretical basis for the reliability, economy and compatibility of EV charging facilities operation under fast charging mode, and provide a basis for optimizing the operation mode and economic benefits of EV charging facilities The optimal scheme produces good economic and social benefits.

\section{Prediction method of load time- space distribution of EV load}

The temporal and spatial distribution of fast charging load of EVs in the transportation network is closely related to the distribution of traffic trips. The following establishes an EV load forecasting model based on the distribution of road network traffic for urban and intercity transportation networks.

\subsection{Prediction of load time-space distribution of EVs on intercity fast charging network}


At present, the inter-city fast charging network covers the backbone transportation network with expressways as the core. Based on the expressway, research the prediction method of time-space distribution of EV load on intercity fast charging network. In order to study the time-space distribution of EV load in the intercity fast charging network, it is first necessary to analyze the travel behavior of EVs and the flow of inbound stations.

\subsection{Road network traffic forecast}

Expressway traffic distribution can generally be combined with expressway OD (O represents the starting point of travel, D represents the travel destination) distribution sentiments in previous years, and the use of elastic algorithms, neural network algorithms, four-stage methods, etc., to obtain the traffic data of the expressway in that year. When calculating the OD flow using the Frett method, an initial OD matrix is required (the OD matrix refers to a matrix composed of the travel volume between any two traffic districts). The OD matrix can often be obtained by investigation.

In this paper, the OD matrix is calculated based on the entrance and exit data of the expressway toll station, combined with the unconstrained center of gravity method, the initial OD matrix is calculated iteratively, and the combined model is used to predict the flow of each entrance and exit. The method of calculating the OD flow distribution of the road network uses the Leit method. The purpose of using the combined forecasting method is to minimize the forecasting error and determine the combined coefficients by establishing a quadratic plan. The analysis of the gray prediction model described above is as follows, suppose the original data series[6]

$$
x^{(0)}=\left[x^{(0)}(1), x^{(0)}(2), x^{(0)}(3) \ldots x^{(0)}(n)\right]
$$

Use 1-AGO to generate a first-order cumulative generating sequence

$$
\left\{\begin{array}{l}
x^{(1)}=\left[x^{(1)}(1), x^{(1)}(2), x^{(1)}(3), \ldots, x^{(1)}(n)\right], \\
x^{(1)}(k)=\sum_{i=1}^{k} x^{(0)}(i), k=1,2,3 \ldots, n
\end{array}\right.
$$

When the sequence $x^{(1)}(k)$ has an approximate exponential growth law, it can be considered that it approximately satisfies the following first-order differential equation, in which assuming that a and $u$ are both known constants, then

$$
\frac{d x^{(1)}}{d t}+a x^{(1)}=u
$$

The formula is

$$
\mathrm{x}^{(1)}(\mathrm{t})=\left[\mathrm{x}^{(0)}-\frac{\mathrm{u}}{\mathrm{a}}\right] \mathrm{e}^{-\mathrm{at}}+\frac{\mathrm{u}}{\mathrm{a}}
$$

The formula is written in discrete form

$$
\mathrm{x}^{1}(\mathrm{k}+1)=\left[\mathrm{x}^{(0)}-\frac{\mathrm{u}}{\mathrm{a}}\right] \mathrm{e}^{-\mathrm{at}}+\frac{\mathrm{u}}{\mathrm{a}}
$$

The prediction model is

$$
\begin{gathered}
x^{0}(k+1)=x^{(3)}(k+1)-x^{(1)}(k)=\left(e^{-a}-\right. \\
\text { 1) }\left[x^{(0)}(1)-\frac{u}{a}\right] e^{-a k}, k=0,1, .
\end{gathered}
$$

The formula is written in continuous form

$$
x^{0}(t)=\left(e^{-a}-1\right)\left[x^{(0)}(1)-\frac{u}{a}\right] e^{-a t}
$$

By establishing the gray model $\operatorname{GM}(1,1)$, it can be seen that the $\operatorname{GM}(1,1)$ can only be used when the original number sequence meets the approximate exponential growth trend. Therefore, when the original number sequence does not meet the above exponential growth trend, use $\operatorname{GM}(1,1)$ usually produces large errors in prediction. When using this model, the constant a can be calculated and evaluated to reflect the extent to which the original data sequence meets the approximate exponential growth trend. Therefore, it is necessary to give the derivation process of solving the constant a.

When the constructed sequence of numbers can satisfy the growth law, the following first obtains the derivative through the first order differential equation[7]

$$
\frac{\mathrm{d}^{2} \mathrm{x}^{(1)}}{\mathrm{dt}^{2}}=-\mathrm{a} \frac{\mathrm{dx^{(1) }}}{\mathrm{dt}}
$$

Due to

$$
\frac{\mathrm{d}^{2} \mathrm{x}^{(1)}}{\mathrm{dt}^{2}}=-\mathrm{a} \frac{\mathrm{dx}(1)}{\mathrm{dt}}, \frac{\mathrm{dx}^{(0)}}{\mathrm{dt}}=\frac{\mathrm{d}^{2} \mathrm{x}^{(0)}}{\mathrm{dt}^{2}}
$$

From the formula

$$
a=-\frac{\frac{d x^{(0)}(t)}{d t}}{x^{(0)}(t)}
$$

In fact, the value of the value a in the $\operatorname{GM}(1,1)$ also reflects to a certain extent whether the original sequence satisfies the approximate exponential growth trend and the extent to which it satisfies the exponential growth trend. That is to say, as a is smaller, the sequence with original data meets the trend of approximate exponential growth better[8].

The values of $x^{(0)}(k)$ and $x^{(0)}(1)$ can be obtained from the formula, and further sorted into:

$$
\mathrm{a}=-\frac{1}{\mathrm{k}-1} \ln \left(\frac{\mathrm{x}^{(0)}(\mathrm{k})}{\mathrm{x}^{(0)}(\mathrm{t})}\right), \mathrm{k}=2,3, \ldots
$$

\subsection{The travel path of EVs}

Traffic distribution is the specific realization form of a certain overall traffic volume, including total time distribution, time ratio distribution, comprehensive distribution and other methods. The time ratio distribution method first distributes the flow to different paths according to the traffic travel rules of the vehicles and their respective traffic travel needs, 
and the traffic distribution knows the time the flow travels on the path, and performs the flow according to the principle of inverse proportion to the time distribution. The shortest path method in the specific implementation form of traffic distribution is simple and straightforward. It considers the shortest path between ODs. This method is based on road crossings such as expressways and routes with fewer entrances and exits. Most travelers will Choose the shortest road as your travel route. Although affected by factors such as weather, road conditions, and the actual construction of charging stations in the service area, it is still the best road selection method[9].

\section{Load forecasting process}

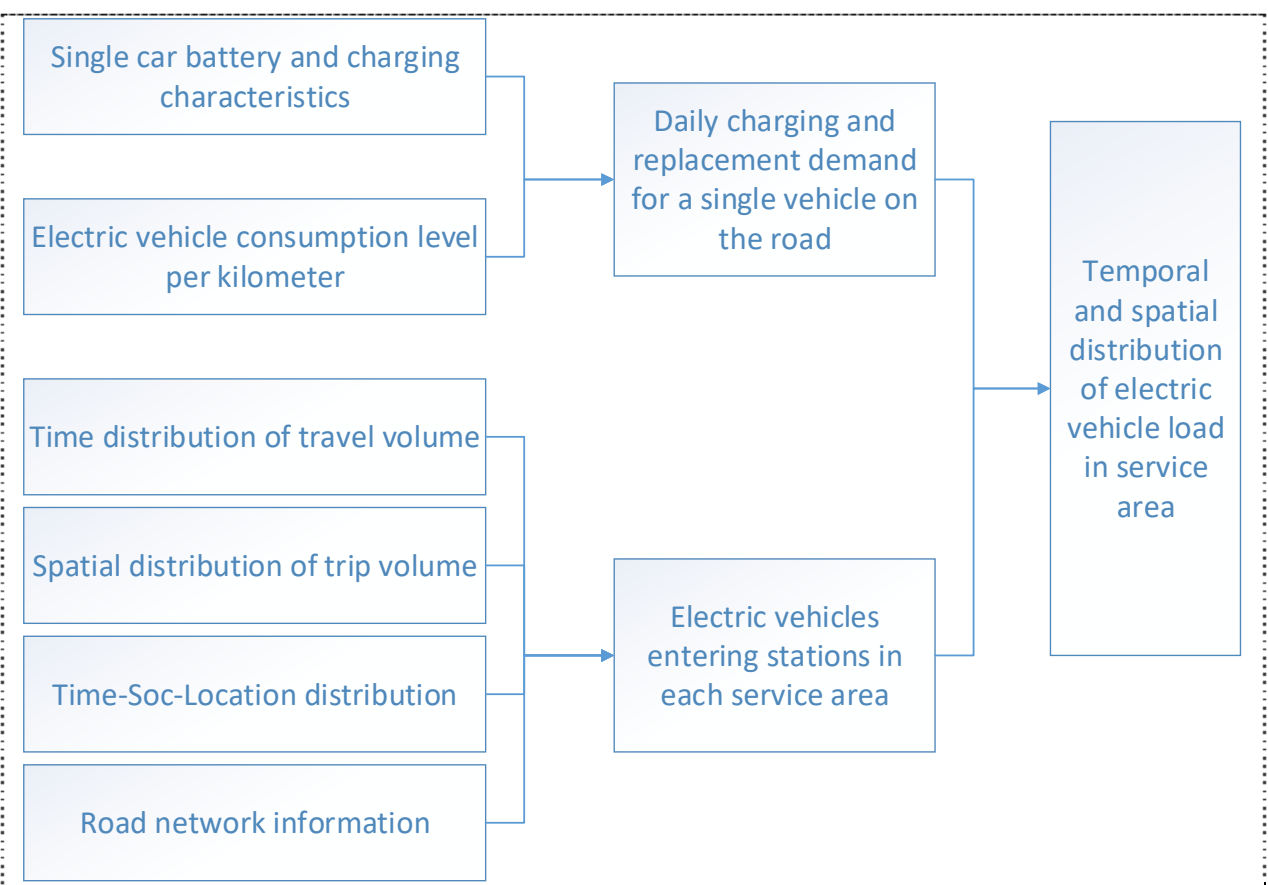

Figure 1. Flowchart of forecasting time and space distribution of EV load on intercity fast charging network

\section{Case analysis}

Calculate the OD matrix based on the entrance and exit data of the expressway toll station, combined with the unconstrained center of gravity method, and calculate the initial OD matrix through iteration. The predicted value of the entrance and exit flow of each expressway toll station is obtained by the combined model, and the OD flow is calculated by the Frett method distributed. In the simulation process, it is assumed that all EVs use fast charging, and their charging power is between 25 and $60 \mathrm{~kW}$. At the same time, it is assumed that the power consumption of EVs per kilometer is the same, which is $0.15 \mathrm{kWh}$. In this simulation model, 30 charging stations in different cities are selected for simulation. Figure 2 shows the time-space distribution of EV charging load:
Considering the time and space distribution of EVs, involving the selection of multiple random variables, the Monte Carlo method is adopted to simulate the time-space distribution of EV load. First, select variables and establish a simulation process. For the simulation of EVs, the parameters involved include departure time, departure location, and initial SOC distribution of EVs. Secondly, determine the probability density function based on actual experience or data fitting. The density function draws a random sample; monitors the remaining power of the battery to determine whether it needs to be charged. If the remaining power of the EV battery is less than $30 \%$ during driving, or the remaining mileage cannot reach each intermediate node on the way to the destination, it is considered that it needs to be charged. Figure 1 is a flowchart of forecasting time-space distribution of EV load on intercity fast charging network[10]. 


\section{Conclusion}

This paper studies the prediction method of the timespace distribution of EV charging demand in the fast charging mode. Based on the expressway, the prediction method of the time-space distribution of EV load in the intercity fast charging network is studied, and a load prediction model of time-space distribution is established. By constructing a prediction method for the time-space distribution of EV charging demand in the fast charging mode, it provides theoretical and methodological support for the research of timesharing and segmented metering and charging strategies for EV fast charging stations, and provides an important reference for the development of EV charging facilities operating cost benefits, economic performance indicators and calculation models under fast charging mode, which are of great significance to promote the popularization and application of EV fast charging modes.

\section{Acknowledgments}

This work was supported by the key technologies research of EVs smart coordinated charging and discharging (KJ19-1-09) of China State Grid Tianjin Electric company.

\section{References}

1. Han Seung Jang, Kuk Yeol Bae \& Dan Keun Sung(2020). Apartment-level electric vehicle charging coordination: peak load reduction and charging payment minimization. 223.

2. Xun Zong \& Xing He(2020). A neurodynamic algorithm to optimize residential demand response problem of plug-in electric vehicle. 4051-11.

3. Jing Zhang, Jie Yan, Yongqian Liu \& Guoliang Lv(2020). Daily electric vehicle charging load profiles considering demographics of vehicle users. 274.

4. M.Kandidayeni, A. Macias \& S. Kelouwani(2020). Investigating the impact of ageing and thermal management of a fuel cell system on energy management strategies. 274.

5. Veeraj V. Shet \& Kiran Jacob(2020). Design of a thermal management system for a battery pack in an electric vehicle using Dymola. 49(5), 26862705.

6. Fucun Li, Hongxia Guo, Zhen Jing \& Xinku Wang(2019). Peak and valley regulation of distribution network with electric vehicles. 2019(16), 2488-2492.

7. Hong Gao, Kai Liu \& Cheng Li(2020). Optimal Location of Fast Charging Stations for Mixed Traffic of Electric Vehicles and Gasoline Vehicles Subject to Elastic Demands. 13(8).

8. Changran He, Guoye Wang, Zhangpeng Gong \& Dongxin $\mathrm{Xu}(2018)$. A Control Algorithm for the
Novel Regenerative-Mechanical Coupled Brake System with by-Wire Based on Multidisciplinary Design Optimization for an Electric Vehicle. 11(9).

9. Umar Hanif Ramadhani, Mahmoud Shepero, Joakim Munkhammar \& Nicholas Etherden(2020). Review of probabilistic load flow approaches for power distribution systems with photovoltaic generation and electric vehicle charging. 120.

10. Constance Crozier \& Malcolm McCulloch(2020). The opportunity for smart charging to mitigate the impact of electric vehicles on transmission and distribution systems. 268. 